\title{
Complicações funcionais do tratamento da instabilidade patelar com reconstrução do ligamento femoropatelar medial com terço medial do tendão patelar com acompanhamento mínimo de 5 anos*
}

\section{Functional Complications of Patellar Instability Treatment with Medial Patellofemoral Ligament Reconstruction with the Medial Third of the Patellar Tendon with a Minimum 5-Year Follow-Up}

\author{
Gilberto Luis Camanho ${ }^{1}$ (1) Riccardo Gomes Gobbi ${ }^{20}$ \\ ${ }^{1}$ Departamento de Ortopedia e Traumatologia, Faculdade de \\ Medicina, Universidade de São Paulo, São Paulo, SP, Brasil \\ ${ }^{2}$ Grupo de Joelho, Instituto de Ortopedia e Traumatologia, Hospital \\ das Clínicas, Faculdade de Medicina, Universidade de São Paulo, São \\ Paulo, SP, Brasil \\ ${ }^{3}$ Instituto Ortopédico Camanho, São Paulo, SP, Brasil
}

\author{
Marta Halasz de Andrade ${ }^{3}$ (1)
}

\begin{abstract}
Endereço para correspondência Riccardo Gomes Gobbi, PhD, Instituto de Ortopedia e Traumatologia, Hospital das Clínicas, Faculdade de Medicina, Universidade de São Paulo, Rua Ovídio Pires de Campos, 333, $3^{\circ}$ andar, Cerqueira César, São Paulo, SP, 05403-010, Brasil (e-mail: r.gobbi@hc.fm.usp.br).
\end{abstract}

Rev Bras Ortop 2022;57(2):308-313.

\section{Resumo \\ Palavras-chave \\ - joelho \\ - luxação patelar \\ - instabilidade articular \\ - ligamentos \\ - recidiva}

Objetivo Avaliar complicações maiores após um mínimo de 5 anos de acompanhamento após luxação aguda ou recidivante da patela tratada com reconstrução do ligamento femoropatelar medial (LFPM) com terço medial do tendão patelar, com ou sem medialização da tuberosidade anterior da tíbia (TAT) associada.

Métodos Um total de 50 pacientes foram incluídos, com acompanhamento mínimo de 5 anos. Os pacientes foram avaliados em relação à ocorrência de complicações como rigidez articular, recidiva de luxação patelar ou instabilidade subjetiva relatada pelos pacientes, e incapacidade de retorno ao nível prévio de atividades físicas.

Resultados O acompanhamento médio foi de 8,9 $\pm 2,6$ anos, com mínimo de 6 e máximo de 15 anos; 64\% dos pacientes eram mulheres, com média de idade de $27 \pm 11,2$ anos; $24 \%$ dos pacientes foram submetidos a osteotomia da TAT para medialização concomitantemente; e $46 \%$ eram casos agudos. Foram constatados apenas 9 maus resultados (18\%), todos decorrentes de recidiva da luxação (12\%) e de queixa de instabilidade subjetiva (6\%), ocorridos entre 36 e 60 meses de
Trabalho desenvolvido no Instituto de Ortopedia e Traumatologia do Hospital das Clínicas da Faculdade de Medicina da Universidade de São Paulo, São Paulo, SP, Brasil. recebido

25 de Maio de 2020

aceito

03 de Novembro de 2020

Publicado on-line

Dezembro 13, 2021
DOI https://doi.org/ 10.1055/s-0041-1729570. ISSN 0102-3616.
(C) 2021. Sociedade Brasileira de Ortopedia e Traumatologia. All rights reserved.

This is an open access article published by Thieme under the terms of the Creative Commons Attribution-NonDerivative-NonCommercial-License, permitting copying and reproduction so long as the original work is given appropriate credit. Contents may not be used for commercial purposes, or adapted, remixed, transformed or built upon. (https://creativecommons.org/ licenses/by-nc-nd/4.0/)

Thieme Revinter Publicações Ltda., Rua do Matoso 170, Rio de Janeiro, RJ, CEP 20270-135, Brazil 


\section{Abstract \\ Keywords \\ - knee \\ - patellar dislocation \\ - joint instability \\ - ligaments \\ - recurrence}

acompanhamento. Não ocorreram outras complicações. Dentre os maus resultados, cinco ocorreram em casos de luxação aguda, e quatro em casos recidivantes, e apenas um havia sido submetido a osteotomia da TAT.

Conclusão A reconstrução do LFPM com terço medial do tendão patelar, associada ou não à osteotomia de medialização da TAT, é uma alternativa no tratamento da instabilidade patelar aguda ou crônica, com falha de apenas 18\% em acompanhamento mínimo de 5 anos. Além disso, é um tratamento seguro, sem apresentar outras complicações.

Objective To evaluate major complications after a minimum of 5 years of follow-up after acute or recurrent patellar dislocation treated with medial patellofemoral ligament (MPFL) reconstruction with the medial third of the patellar tendon, with or without associated medialization of the tibial anterior tuberosity (TAT).

Methods A total of 50 patients were included, with a minimum follow-up of 5 years. The patients were evaluated regarding complications such as joint stiffness, recurrence of patellar dislocation, subjective instability reported by patients, and inability to return to the previous level of physical activity.

Results The mean follow-up was of $8.9 \pm 2.6$ years, with a minimum of 6 and maximum of 15 years; $64 \%$ of the patients were women, with a mean age of $27 \pm 11.2$ years old; $24 \%$ were submitted to TAT osteotomy for simultaneous medialization; and $46 \%$ were acute cases. Only 9 poor results (18\%) were found, all resulting from recurrence of dislocation (12\%) and complaint of subjective instability (6\%) at between 36 and 60 months of follow-up. No other complications occurred. Among the poor results, five occurred in cases of acute dislocation, and four in recurrent cases, and only one had undergone TAT osteotomy.

Conclusion Reconstruction of the MPFL with the medial third of the patellar tendon, associated or not with TAT medialization osteotomy, is an alternative in the treatment of acute or chronic patellar instability, with a failure rate of only $18 \%$ in at least 5 years of follow-up. In addition, it is safe treatment, that does not present other complications.

\section{Introdução}

A instabilidade femoropatelar é uma queixa bastante desafiadora na prática clínica. Contudo, suas manifestações maiores, a luxação femoropatelar aguda e recidivante, são pouco frequentes. A luxação aguda ocorre em apenas entre 2 a $3 \%$ dos traumas de joelho, e a falha de seu reconhecimento é uma das causas mais frequentes de erro diagnóstico nos casos de lesões agudas do joelho. ${ }^{1}$

Os casos agudos de luxação femoropatelar tradicionalmente recebiam tratamento conservador até o conhecimento do ligamento femoropatelar medial (LFPM), que culminou numa importante mudança dos princípios de tratamento. Vários autores sugerem o reparo ou a reconstrução deste ligamento nos casos agudos, evitando as recidivas da primoluxação que chegam a $>50 \%^{2-5}$

Os fatores predisponentes sempre foram muito valorizados, especialmente nos casos crônicos, sendo que o chamado "menu à la carte" dominou a linha terapêutica. Hoje, após o conhecimento e ampla assimilação do LFPM desde o início dos anos 2000, a reconstrução do LFPM assumiu um papel fundamental no tratamento dos casos de instabilidades patelares. ${ }^{6}$
Nosso grupo reconheceu e estudou o LFPM $^{7}$ e passou inicialmente a repará-lo nos casos agudos e em alguns casos crônicos. ${ }^{2}$ Devido a algumas falhas no reparo, passamos a reconstruí-lo nos casos agudos e crônicos. Este estudo culminou no desenvolvimento da técnica utilizando o terço medial do tendão patelar como enxerto, ${ }^{8}$ e em sua validação em casos agudos em comparação com tratamento conservador sem nenhum caso de recidiva da luxação em 38 meses de acompanhamento. 3,9

O presente trabalho tem a intenção de descrever complicações maiores e recidivas tardias após um mínimo de 5 anos de acompanhamento em pacientes que, após a luxação aguda ou recidivante da patela, tiveram o LFPM reconstruído com o terço medial do tendão patelar, com ou sem medialização da tuberosidade anterior da tíbia (TAT) associada.

\section{Métodos}

Foram incluídos pacientes tratados pelos autores entre 2005 e 2015 que apresentavam instabilidade femoropatelar e foram submetidos a cirurgia de reconstrução do LFPM com enxerto do terço medial do tendão patelar e tiveram mínimo 5 anos de acompanhamento. Todos os pacientes seguiram o protocolo 


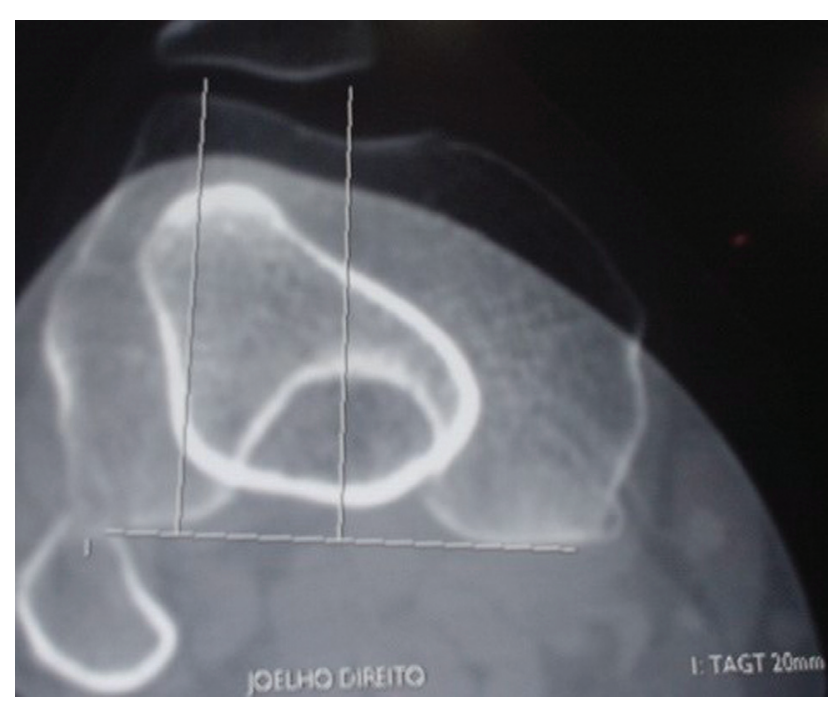

Fig. 1 Corte axial de tomografia computadorizada com medida da distância da tuberosidade anterior da tíbia ao sulco troclear de $20 \mathrm{~mm}$.

institucional de reabilitação realizado pela mesma equipe. 0 diagnóstico de instabilidade patelar era confirmado após a avaliação da história, da realização de exame clínico sugestivo realizado por médico experiente em cirurgia do joelho, e por imagem compatível (radiografia e ressonância magnética [RM]).

A luxação foi classificada no presente trabalho como aguda quando tratada nos primeiros 90 dias da primoluxação traumática, e crônica após este período ou quando era recidivante ( $\geq$ 2episódios de luxação completa da patela).

Todos os pacientes, previamente à realização da cirurgia, foram avaliados através de radiografias e tomografia computadorizada (TC) seguindo os preceitos de Dejour et al., ${ }^{10,11} \mathrm{e}$ também por RM de acordo com a experiência do nosso grupo. $^{12-15}$ Foram avaliados a altura da patela, displasia troclear, o alinhamento eixo anatômico, a distância da tuberosidade anterior da tíbia ao sulco troclear (TAGT) e lesões condrais. Foram incluídos apenas pacientes que não possuíam indicação para abaixamento da patela (índice Caton-Des- champs $>1,3$ ), trocleoplastia (displasia troclear tipo B e D com bump $>5 \mathrm{~mm}$ ), tratamento cirúrgico de lesão condral ou osteotomias corretivas para eixo. Como procedimento associado à reconstrução do LFPM, foi aceita inclusão apenas de medialização da TAT pela cirurgia de Elmslie-Trillat ${ }^{16}$ nos casos de TAGT $\geq 20 \mathrm{~mm}$ ( - Figura $\mathbf{1}$ ).

Para a reconstrução do LPFM utilizou-se $0,5 \mathrm{~cm}$ da parte medial do tendão patelar, com desinserção tibial e mantendo inserção na patela, com descolamento subperiostal até o terço proximal da patela na posição anatômica do ligamento. ${ }^{8,17}$ O enxerto preparado é então fixado no côndilo femoral medial, em um ponto proximal e posterior ao epicôndilo medial, entre este e o tubérculo dos adutores, ${ }^{17,18}$ como mostrado na - Figura 2.

Para os pacientes com indicação da técnica de ElmslieTrillat apud Gomes et al., ${ }^{19}$ a medialização da TAT foi suficiente para corrigir a TAGT para um valor entre 10 e $15 \mathrm{~mm}$, sendo realizada fixação com parafuso esponjoso. Com o joelho em $90^{\circ}$ de flexão, era checado se a patela se apresentava centrada aos côndilos e se a TAT se encontrava alinhada com o sulco troclear para definir a posição final da correção. A reconstrução do LFPM seguia exatamente os mesmos passos descritos acima.

Os pacientes eram orientados a utilizar imobilizador por 1 semana e muletas por 15 dias após o procedimento. A fisioterapia iniciou-se nos primeiros dias de pós-operatório e estendeu-se por 2 a 3 meses. O objetivo era o ganho total de movimento e a recuperação da força muscular.

O acompanhamento mínimo foi de 5 anos. A ocorrência de complicações foi determinada na última consulta do paciente, a qual define o tempo final de acompanhamento.

Os pacientes foram divididos em dois grupos a critério dos autores de acordo com a presença das seguintes complicações maiores:

- Bom resultado: retorno ao mesmo nível de atividade física anterior à luxação e ausência de novas luxações ou de queixa de instabilidade subjetiva;
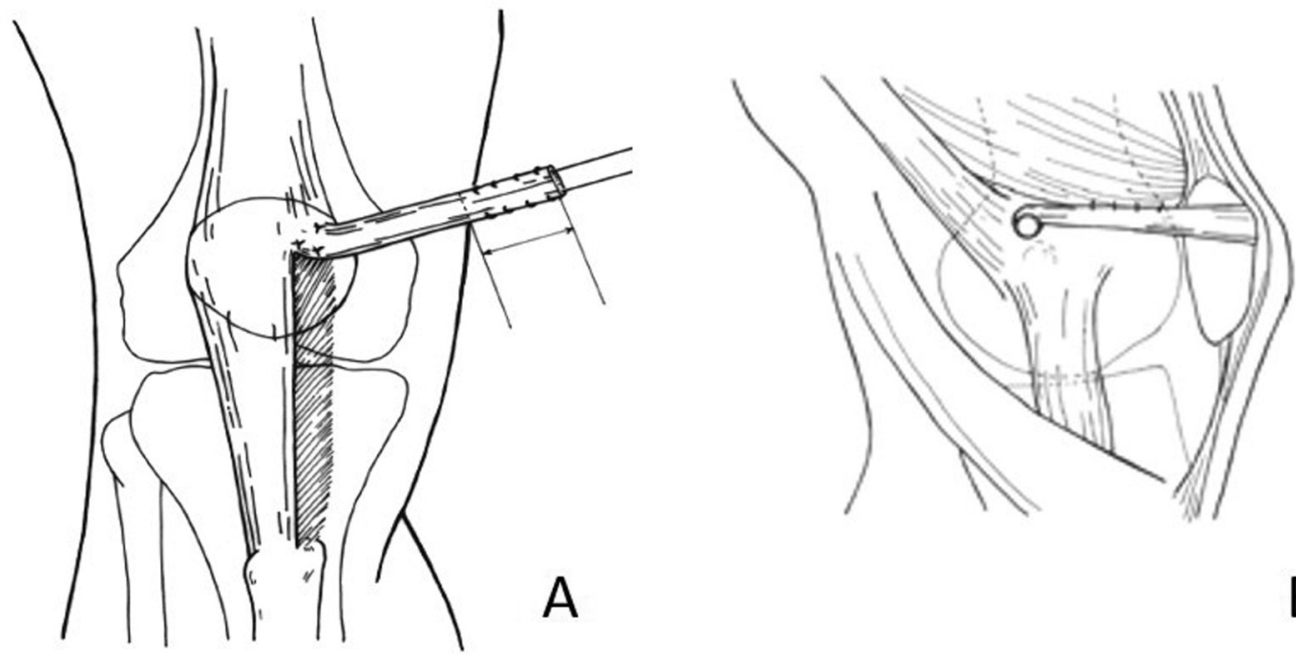

Fig. 2 A - Preparo de $0,5 \mathrm{~cm}$ medial do tendão patelar até a transição entre o terço proximal e o médio da patela. B - Fixação do enxerto entre o epicôndilo medial e o tubérculo dos adutores. 


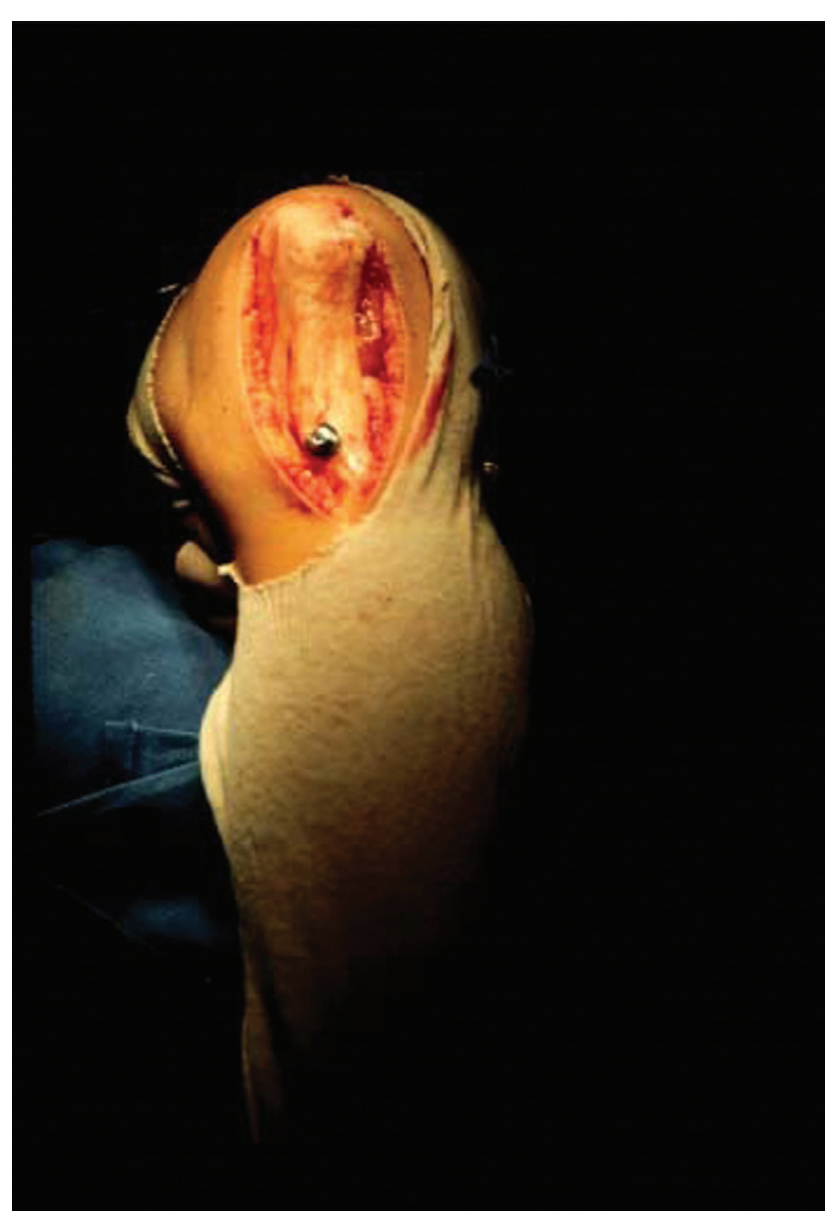

Fig. 3 Medialização da tuberosidade anterior da tíbia fixada com um parafuso esponjoso (segundo técnica de Elmslie-Trillat).

- Mau resultado: incapacidade de retornar aos níveis prévios de atividade física, limitação articular (rigidez com perda $>10^{\circ}$ da amplitude de movimento do joelho), ocorrência de novos episódios de luxação (recidiva), ou instabilidade subjetiva sintomática relatada pelo paciente.

\section{Resultados}

Foram incluídos 50 pacientes. Destes, 23 (46\%) eram casos agudos e 27 (54\%) eram casos crônicos. 0 sexo feminino foi o mais frequentemente acometido, com 32 (64\%) pacientes, sendo o lado direito acometido em 26 (52\%) pacientes.

A maior parte dos pacientes tinha $<30$ anos, com média de 27 anos $\pm 11,2$ anos, mínimo de 14 e máximo de 58 anos, e com a seguinte distribuição:

- <20 anos: 18 casos (36\%);

- De 20 a 30 anos: 14 casos (28\%);

- De 30 a 40 anos: 13 casos (26\%);

- > de 40 anos: 5 casos (10\%).

Em relação ao tempo de acompanhamento:

- entre 5 e 10 anos: 34 casos (68\%);

- mais de 10 anos: 16 casos (32\%).

Foram avaliados os fatores predisponentes (patela alta, tróclea displásica, inclinação lateral patelar e eixo anatômico em valgo). Entre os 23 pacientes com episódios agudos, 15 (70\%) tinham ao menos um fator predisponente. A patela alta (Caton-Deschamps entre 1,2 e 1,3) foi o mais frequente e ocorreu em 8 pacientes. Entre os 27 casos crônicos, apenas 2 não possuíam um fator predisponente. A patela alta foi o mais frequente e ocorreu em 13 pacientes. Não tivemos nenhum paciente com valgo $>15^{\circ}$.

Quanto à medialização da TAT, o realinhamento distal foi feito em $5(21,7 \%)$ casos entre os agudos e em 7 (26\%) casos entre os crônicos.

A casuística global apresentou 41 resultados bons (82\%) e 9 maus (18\%). Os pacientes jovens ( $<30$ anos) tiveram $78 \%$ dos maus resultados, com 3 casos de mau resultado até 20 anos (16,7\% da faixa etária), 4 entre 20 e 30 anos (28,6\% da faixa etária), sendo apenas 2 nos pacientes $>30$ anos ( $11 \%$ da faixa etária).

Quanto ao momento da cirurgia, 5 (55\%) dos maus resultados foram em pacientes com luxação aguda ( $21,7 \%$ destes), contra 4 (45\%) em casos crônicos (14,8\% destes).

O principal critério de mau resultado foi a ocorrência de um novo episódio de luxação (6 dos 9 casos; 67\%), todos ocorrendo entre o $3^{\circ}$ e o $5^{\circ}$ ano de acompanhamento. Destes, 3 pacientes eram casos agudos, o que equivale a $50 \%$ das recidivas da instabilidade na casuística. Os outros três casos com mau resultado consistiram em queixas de instabilidade subjetiva, sem manifestação de luxação completa. Entre os 12 pacientes submetidos ao realinhamento distal, e apenas 1 caso (8\%) crônico apresentou mau resultado devido a nova luxação. Não houve nenhum caso de rigidez ou de incapacidade de retorno às atividades prévias que não fosse a recidiva da instabilidade.

Os dados encontram-se resumidos na - Tabela 1, e os casos separados em bom e mau resultado na - Tabela 2.

Tabela 1 Resumo da casuística

\begin{tabular}{|c|c|c|}
\hline \multirow[t]{2}{*}{ GÊNERO } & Masculino & $18(36 \%)$ \\
\hline & Feminino & $32(64 \%)$ \\
\hline \multirow[t]{2}{*}{ LADO } & Direito & 26 (52\%) \\
\hline & Esquerdo & $24(48 \%)$ \\
\hline IDADE & & $\begin{array}{l}27 \pm 11,2 \text { anos } \\
\text { mín-máx } 14-58\end{array}$ \\
\hline ACOMPANHAMENTO & & $\begin{array}{l}8,9 \pm 2,6 \text { anos } \\
\text { mín-máx 6-15 }\end{array}$ \\
\hline OSTEOTOMIA TAT & & $12(24 \%)$ \\
\hline \multirow[t]{2}{*}{ TIPO INSTABILIDADE } & Aguda & $23(46 \%)$ \\
\hline & Crônica & 27 (54\%) \\
\hline \multirow[t]{2}{*}{ RESULTADO } & Bom & $41(82 \%)$ \\
\hline & Mau & $9(18 \%)$ \\
\hline \multirow[t]{2}{*}{$\begin{array}{l}\text { MOTIVO MAU } \\
\text { RESULTADO }\end{array}$} & Recidiva & $\begin{array}{l}6 \text { (67\% dos maus, } \\
12 \% \text { do total) }\end{array}$ \\
\hline & $\begin{array}{l}\text { Instabilidade } \\
\text { subjetiva }\end{array}$ & $\begin{array}{l}3 \text { ( } 33 \% \text { dos maus, } \\
6 \% \text { do total) }\end{array}$ \\
\hline
\end{tabular}

Abreviação: TAT, tuberosidade anterior da tíbia. 
312 Complicações funcionais do tratamento da instabilidade patelar Camanho et al.

Tabela 2 Dados separados por bom e mau resultado

\begin{tabular}{|l|l|l|l|}
\hline & & Bom resultado $(\boldsymbol{n}=\mathbf{4 1})$ & Mau resultado $(\boldsymbol{n}=\mathbf{9})$ \\
\hline \multirow{2}{*}{ GÊNERO } & Masculino & $14(34 \%)$ & $4(44 \%)$ \\
\cline { 2 - 4 } & Feminino & $27(66 \%)$ & $5(56 \%)$ \\
\hline \multirow{2}{*}{ LADO } & Direito & $22(54 \%)$ & $4(44 \%)$ \\
\cline { 2 - 4 } & Esquerdo & $19(46 \%)$ & $5(56 \%)$ \\
\hline IDADE (anos) & & $27,2 \pm 11,3$ & $26,1 \pm 11,1$ \\
\hline ACOMPANHAMENTO (anos) & & $8,8 \pm 2,5$ & $8,9 \pm 3$ \\
\hline OSTEOTOMIA TAT $(n=12)$ & & $11(92 \%$ das osteotomias) & $1(8 \%$ das osteotomias) \\
\hline \multirow{2}{*}{ TIPO INSTABILIDADE } & Aguda & $18(44 \%)$ & $5(56 \%)$ \\
\cline { 2 - 4 } & Crônica & $23(56 \%)$ & $4(44 \%)$ \\
\hline MOTIVO MAU RESULTADO & & & $\begin{array}{l}\text { Nova luxação: } 6(67 \%) ; \\
\text { Instabilidade subjetiva: } 3(33 \%)\end{array}$ \\
\hline
\end{tabular}

Abreviação: TAT, tuberosidade anterior da tíbia.

\section{Discussão}

A principal importância do presente trabalho é descrever as complicações dos pacientes agudos e crônicos com luxação femoropatelar, tratados pela reconstrução do LFPM com o terço médio do tendão patelar, após um mínimo de 5 anos de acompanhamento.

A discussão quanto à indicação cirúrgica ou conservadora da luxação aguda da patela foi extensa nos trabalhos prévios do grupo, nos quais demonstramos percentuais de falha $>35$ a 50\% em até 2 anos nos pacientes que tiveram suas luxações tratadas conservadoramente. ${ }^{2,3}$ Similarmente, Maenpää et al. ${ }^{20}$ demonstram percentuais $>50 \%$ de recidiva da luxação em 100 pacientes tratados de forma conservadora após uma média de 13 anos de acompanhamento, índice muito maior do que as falhas cirúrgicas ocorridas na presente casuística (18\%), com acompanhamento médio de 9 anos.

Fatores predisponentes são muito prevalentes em pacientes com instabilidade patelar. ${ }^{10}$ Dos 23 pacientes com quadro agudo, 15 tinham fatores predisponentes, sendo 8 casos de patela alta. Dos 27 casos crônicos, 25 tinham algum fator predisponente, sendo 13 com patela alta. Nenhum caso de distalização foi incluído; nenhum dos pacientes apresentava índice de Caton-Deschamps $>1,3$.

0 realinhamento distal feito em 12 pacientes não trouxe nenhum problema adverso. Uma porcentagem discretamente maior de casos crônicos foi submetida ao realinhamento distal. Acreditamos que este fato era esperado, uma vez que casos que desenvolvem instabilidade recidivante/crônica tendem a possuir um perfil anatômico mais displásico. ${ }^{10}$

A distribuição quanto ao gênero, lado e idade foi semelhante às casuísticas estudadas anteriormente pelo grupo, sendo representativa da população portadora de instabilidade patelar.

Os resultados encontrados reafirmam nossa prática de indicação da reconstrução do LFPM, associada ou não ao realinhamento distal, como efetiva no tratamento da luxação da patela, seja ela aguda ou recidivante. Os resultados da reconstrução do LFPM no tratamento das luxações agudas com a técnica descrita já foram publicados pelo nosso grupo, com ausência de episódios de luxação após acompanhamento de 38 meses. $^{3}$ Achados similares são encontrados em vários estudos de luxações agudas, já incluídos em meta-análises. ${ }^{5} \mathrm{~A}$ ocorrência de recidiva da instabilidade após esse período (todos nossos casos ocorreram após 36 meses), chama a atenção para o fato de que o resultado do tratamento da instabilidade patelar deve ser avaliado a longo prazo, pois os pacientes apresentam variados graus de displasia, muitas vezes limítrofes e sem indicação de correção, mas que mantém um risco de instabilidade maior do que o da população normal. Neste sentido, os pacientes com instabilidade patelar merecem atenção por muito mais tempo que um paciente submetido a reconstrução do ligamento cruzado anterior por exemplo.

Em relação ao tratamento das instabilidades crônicas/recidivantes, poucos estudos comparativos são encontrados, sendo que a maioria dos dados provém de séries de casos. Vavalle et al. ${ }^{21}$ publicaram 16 casos de reconstrução do LFPM com bons resultados após 38 meses de acompanhamento em pacientes portadores de luxação femoropatelar crônica. Os autores utilizaram o tendão quadricipital como enxerto para reconstrução. Não houve casos de novas luxações na série relatada. ${ }^{21}$ Slenker et al. ${ }^{22}$ estudaram 35 pacientes com instabilidade femoropatelar crônica com episódios de luxação, tratados pela reparação do LFPM, feita com aloenxerto em 23 casos e com tendão da pata de ganso em 12. Os autores obtiveram bons resultados após um acompanhamento médio de 21 meses, com melhora do índice de Kujala de 49 no préoperatório para 89,5 no pós-operatório. ${ }^{22}$ Uma revisão sistemática recente incluiu apenas cinco estudos de instabilidade recidivante, concluindo apenas que a reconstrução do LFPM apresenta escores clínicos superiores em relação à cirurgia de plicatura medial. ${ }^{23}$

Não fizemos uma avaliação numérica de escores funcionais dos resultados, utilizando métodos quantitativos, como o de Kujala et al. ${ }^{24}$ Essa avaliação já foi publicada em outros trabalhos, em comparação de métodos de tratamento, cirúrgico e conservador; os resultados foram muito melhores nos pacientes submetidos a tratamento cirúrgico pela reparação 
ou pela reconstrução do LFPM. Nossos estudos prévios mostraram uma média do Kujala de 89 pontos após 3 anos de acompanhamento, bastante similar ao restante da literatura e de outras técnicas e enxertos. ${ }^{25} \mathrm{~A}$ funcionalidade dos pacientes se manteve nos anos seguintes, exceto naqueles que sofreram recidiva da instabilidade. Devemos realçar que pacientes portadores de instabilidade femoropatelar recidivante, em geral, não se dedicam a prática esportiva de alto nível pela própria limitação causada pela doença, portanto as operações devolveram estes pacientes a sua atividade anterior às luxações, porém sem a instabilidade, na maioria dos casos. O objetivo aqui é descrever, em um tempo de acompanhamento maior, a ocorrência de falhas importantes como recidiva da luxação. Fica evidenciada a necessidade de acompanhamento de longo prazo pelo fato da ocorrência das recidivas ser mais tardia.

Outra limitação do presente estudo é não avaliar a evolução de degeneração da articulação femoropatelar. São necessários estudos de longo prazo para verificarmos a ocorrência de artrose nestes pacientes, definindo o risco de cada procedimento corretivo e de cada alteração anatômica não corrigida evoluir para degeneração.

\section{Conclusão}

A reconstrução do LFPM com terço medial do tendão patelar, associada ou não à osteotomia de medialização da TAT, é uma alternativa no tratamento da instabilidade patelar aguda ou crônica, com falha de apenas $18 \%$ em acompanhamento mínimo de 5 anos. Além disso, é um tratamento seguro, sem apresentar outras complicações.

\section{Suporte Financeiro}

Não houve suporte financeiro de fontes públicas, comerciais, ou sem fins lucrativos.

\section{Conflito de Interesses}

Os autores declaram não haver conflito de interesses.

\section{Referências}

1 Stefancin JJ, Parker RD. First-time traumatic patellar dislocation: a systematic review. Clin Orthop Relat Res 2007;455(455):93-101

2 Camanho GL, Viegas AdeC, Bitar AC, Demange MK, Hernandez AJ. Conservative versus surgical treatment for repair of the medial patellofemoral ligament in acute dislocations of the patella. Arthroscopy 2009;25(06):620-625

3 Bitar AC, Demange MK, D'Elia CO, Camanho GL. Traumatic patellar dislocation: nonoperative treatment compared with MPFL reconstruction using patellar tendon. Am J Sports Med 2012;40 (01):114-122

4 Sillanpää PJ, Mattila VM, Mäenpää H, Kiuru M, Visuri T, Pihlajamäki H. Treatment with and without initial stabilizing surgery for primary traumatic patellar dislocation. A prospective randomized study. J Bone Joint Surg Am 2009;91(02):263-273

5 Pagliazzi G, Napoli F, Previtali D, Filardo G, Zaffagnini S, Candrian C. A Meta-analysis of Surgical Versus Nonsurgical Treatment of Primary Patella Dislocation. Arthroscopy 2019;35(08):2469-2481

6 Arendt EA, Dejour D. Patella instability: building bridges across the ocean a historic review. Knee Surg Sports Traumatol Arthrosc 2013;21(02):279-293
7 Camanho GL, Viegas AC. Estudo anatômico e artroscópico do ligamento femoropatelar medial. Acta Ortop Bras 2003;11(03):145-149

8 Camanho GL, Bitar AC, Hernandez AJ, Olivi R. Medial patellofemoral ligament reconstruction: a novel technique using the patellar ligament. Arthroscopy 2007;23(01):108.e1-108.e4

9 Bitar AC, D’Elia CO, Demange MK, Viegas AC, Camanho GL. Randomized prospective study on traumatic patellar dislocation: conservative treatment versus reconstruction of the medial patellofemoral ligament using the patellar tendon, with a minimum of two years of follow-up. Rev Bras Ortop 2015;46(06): 675-683

10 Dejour H, Walch G, Nove-Josserand L, Guier C. Factors of patellar instability: an anatomic radiographic study. Knee Surg Sports Traumatol Arthrosc 1994;2(01):19-26

11 Gobbi RG, Demange MK, de Ávila LFR, et al. Patellar tracking after isolated medial patellofemoral ligament reconstruction: dynamic evaluation using computed tomography. Knee Surg Sports Traumatol Arthrosc 2017;25(10):3197-3205

12 Gobbi RG, Hinckel BB, Teixeira PRL, et al. The Vastus Medialis Insertion Is More Proximal and Medial in Patients With Patellar Instability: A Magnetic Resonance Imaging Case-Control Study. Orthop J Sports Med 2019;7(12):2325967119880846

13 Hinckel BB, Gobbi RG, Kihara Filho EN, et al. Why are bone and soft tissue measurements of the TT-TG distance on MRI different in patients with patellar instability? Knee Surg Sports Traumatol Arthrosc 2017;25(10):3053-3060

14 Hinckel BB, Gobbi RG, Kihara Filho EN, Demange MK, Pécora JR, Camanho GL. Patellar Tendon-Trochlear Groove Angle Measurement: A New Method for Patellofemoral Rotational Analyses. Orthop J Sports Med 2015;3(09):2325967115601031

15 Hinckel BB, Gobbi RG, Filho EN, et al. Are the osseous and tendinouscartilaginous tibial tuberosity-trochlear groove distances the same on CT and MRI? Skeletal Radiol 2015;44(08):1085-1093

16 Trillat AD, Dejour H, Couette A. Diagnostic et traitement des subluxations récidivantes de la rotule. Rev Chir Orthop Repar Appar Mot 1964;50:813-824

17 Hinckel BB, Gobbi RG, Demange MK, et al. Medial Patellofemoral Ligament, Medial Patellotibial Ligament, and Medial Patellomeniscal Ligament: Anatomic, Histologic, Radiographic, and Biomechanical Study. Arthroscopy 2017;33(10):1862-1873

18 Gobbi RG, Pereira CA, Sadigursky D, et al. Evaluation of the isometry of different points of the patella and femur for medial patellofemoral ligament reconstruction. Clin Biomech (Bristol, Avon) 2016;38(01):8-12

19 Gomes JL, Sanhudo JA, Marczyk LR, Guerra M, Essaca PM. Avaliação a longo prazo da instabilidade femoropatelar tratada pela técnica de Elmslie-Trillat. Rev Bras Ortop 1996;31(07):595-599

20 Mäenpää H, Lehto MU. Patellar dislocation. The long-term results of nonoperative management in 100 patients. Am J Sports Med 1997;25(02):213-217

21 Vavalle G, Capozzi M. Isolated reconstruction of the medial patellofemoral ligament with autologous quadriceps tendon. J Orthop Traumatol 2016;17(02):155-162

22 Slenker NR, Tucker BS, Pepe MD, Marchetto PA, Cohen SB. Short-/ intermediate-term outcomes after medial patellofemoral ligament reconstruction in the treatment of chronic lateral patellofemoral instability. Phys Sportsmed 2013;41(02):26-33

23 Lee DY, Park YJ, Song SY, Hwang SC, Park JS, Kang DG. Which Technique Is Better for Treating Patellar Dislocation? A Systematic Review and Meta-analysis. Arthroscopy 2018;34(11):3082-3093.e1

24 Kujala UM, Jaakkola LH, Koskinen SK, Taimela S, Hurme M, Nelimarkka O. Scoring of patellofemoral disorders. Arthroscopy 1993;9(02):159-163

25 Buckens CF, Saris DB. Reconstruction of the medial patellofemoral ligament for treatment of patellofemoral instability: a systematic review. Am J Sports Med 2010;38(01):181-188 